\title{
Statistical t-test assessment on performance of power system stabilizer for oscillation damping
}

\author{
Wai Myat Thu', Kyaw Myo Lin² \\ ${ }^{1}$ Department of Electrical Power Engineering, Technological University (Thanlyin), Yangon, Myanmar \\ ${ }^{2}$ Department of Electrical Power Engineering, Pyay Technological University, Pyay, Myanmar
}

\begin{tabular}{l} 
Article Info \\
\hline Article history: \\
Received Apr 4, 2021 \\
Revised May 27, 2021 \\
Accepted Jun 15, 2021 \\
\hline
\end{tabular}

Keywords:

Damping ratio

Hypothesis

MEPE test system

PSS

Statistical t-test

\begin{abstract}
The statistical operation is necessary to validate the analytical procedure since the most important common operation is the comparison of result data to quantify accuracy and precision. In this paper, a statistical process is proposed to ensure the impact of power system stabilizers (PSSs) in 202-bus, 40-machine MEPE test system. The eigenvalue analysis is utilized for detecting the lowest damping modes and evaluating the damping ratio of system oscillation, and these modes are applied as input for statistical analysis. A t-test analysis was performed on the difference of damping performance by proposed designed PSS, tuned PSS, and without PSS to support the hypothesis. The t-test results validated that the MEPE test system with designed PSS is most applicable for the improvement of oscillation damping in the power system.
\end{abstract}

This is an open access article under the CC BY-SA license.

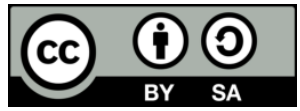

\section{Corresponding Author:}

Wai Myat Thu

Department of Electrical Power Engineering

Technological University (Thanlyin)

Thanlyin city, 4 miles far from Kyauk-Tan city, Yangon Division, Myanmar

Email: waimyatthu@gmail.com

\section{INTRODUCTION}

Statistics is widely used in the research study. In recent years, the application of the statistical test in research activities has increased dramatically in every science [1]. Therefore, this paper presents the use of a statistical tool that evaluates the power system stabilizer (PSS) impacts on the power system. Today, most plants in the interconnected system are realized by AVR and PSS combination [2]. The development of a control system including PSS is necessary to eliminate the power system oscillation to keep stability [3]. Therefore, the PSSs are installed for assisting the automatic voltage regulator (AVR) of generating station in the Myanmar national grid test system (MEPE test system) to damp out oscillation and stability of the existing network [4]. Moreover, PSS parameters are needed to be designed to get the desired oscillation damping [5]. When activating the optimal designed PSSs on the excitation system of some of the dominant generating units get the higher damping in system oscillation [6]. For the appropriate design and to check the robustness of PSS, it is essential to analyze from a statistical point of view to confirm or validate the parameters.

The main goal of this paper is to confirm the differences between these PSS performances that give the better damping oscillation of the MEPE network using statistical hypothesis testing. Test of statistical hypothesis to evaluate two sets of data are well appropriate and determine are different or not, even the sets have significant standard deviations different [7]. The t-test, one of the hypothesis tests has been used in this research. The p-value and t-value are calculated. The results indicate the best possibilities for the application of designed PSSs in oscillation damping. 
The rest of the paper is organized as follows; section 2 presents an overview of the application t-test in research work, section 3 briefly explains the concepts of hypothesis testing, The data analysis for the proposed statistical $t$ test on the damping rate of different type PSS is discussed in section 4 while the probability results of t-test for using PSSs are discussed in section 5. Finally, conclusions are given in section 6.

\section{STATISTICAL t-TEST IN RESEARCH STUDY}

Common statistical tests are applied to evaluate method comparison. As a statistical approach, the student's t-test is often employed to analyze whether the mean value of the normal distribution data from an independent sample is consistent with or departs significantly from the null hypothesis [8].

\subsection{Student t-test}

The $t$ distribution is similar to the standard normal distribution in that it is bell-shaped, symmetric about the mean, the mean, median, and mode are equal to 0 and are located at the center of the distribution, and that the curve never touches the $\mathrm{x}$-axis. The difference with the standard normal distribution is that the variance is greater than 1 . The $t$ distribution is a family of curves based on the degrees of freedom, which is a number related to sample size. When using the t-test to compare two means, it must be determined what are the samples dependent or independent. To test the means difference, independent t-test is employed. The formula of t-test [9] is (1).

$$
t=\frac{\bar{X}-\bar{Y}}{\sqrt{\frac{s_{x}^{2}}{n_{x}}+\frac{s_{y}^{2}}{n_{y}}}}
$$

where, the freedom degree is equal to the smaller of $n_{x}-1$ and $n_{y}-1, \bar{X}-\bar{Y}$ is the observed difference between means of sample and the expected value, $\mu_{1}-\mu_{2}$ is equal to zero when no difference in population means. In (1), $n_{x}$ and $n_{y}$ are the number of samples on each of the data sets $X$ and $Y . \bar{X}, \bar{Y}, S_{x}^{2}$ and $S_{y}^{2}$ are the sample means and sample variances of sets X and Y, respectively. They are calculated as (2), (3), (4), and (5).

$$
\begin{aligned}
& \bar{X}=\frac{\sum_{i=1}^{n} X_{i}}{n_{x}} \\
& \bar{Y}=\frac{\sum_{i=1}^{n} Y_{i}}{n_{y}} \\
& S_{x}^{2}=\frac{\sum_{i=1}^{n}\left(X_{i}-\bar{X}\right)^{2}}{n_{x}-1} \\
& S_{y}^{2}=\frac{\sum_{i=1}^{n}\left(Y_{i}-\bar{Y}\right)^{2}}{n_{y}-1}
\end{aligned}
$$

In statistics, the Pearson correlation coefficient, $(r)$ is needed to calculate. This coefficient is the covariance of the two variables divided by the product of their standard deviations and it is obtained as (6).

$$
r_{\mathrm{xy}}=\frac{\sum_{i=1}^{n}\left(\left(X_{i}-\bar{X}\right) \cdot\left(Y_{i}-\bar{Y}\right)\right)}{\sqrt{\sum_{i=1}^{n}\left(X_{i}-\bar{X}\right)^{2}} \sqrt{\sum_{i=1}^{n}\left(Y_{i}-\bar{Y}\right)^{2}}}
$$

where, $\mathrm{n}$ is the sample size of data and $\mathrm{X}_{\mathrm{i}}$, and $\mathrm{Y}_{\mathrm{i}}$ are the individual sample data. The correlation coefficient ranges from -1 to 1 [10]. The values of $\mathrm{r}$ close to -1 or 1 indicate a strong linear relationship between two samples.

\subsection{Confident interval and probability in statistics}

The confidence level reflects the percent of confidence that the true mean difference, $\mu_{\mathrm{D}}$ is between the confidence interval limits. Typical confidence levels are $90 \%, 95 \%$, and $99 \%$, with $95 \%$ being the most common. It is desired to construct a $100(1-\alpha)$ percent confidence interval for $\mu_{\mathrm{D}}$. In which, $\alpha$, is the significance level used in the hypothesis tests. A value of 0.05 is most commonly used, but $0.1,0.025,0.01$, 
and other values are sometimes used. Typical values range from 0.001 to 0.20 . The p-value is the probability that the test statistic will take a value at least as extreme as the observed value, assuming that the null hypothesis is true. If the p-value is less than the prescribed $\alpha$, in this case, $\alpha=0.05$, the null hypothesis is rejected [9].

\section{HYPOTHESIS TESTING}

A hypothesis test is a statistical way to reject (or to accept) a research hypothesis within a certain degree of confidence. It provides a way of quickly assessing the strength of evidence for a difference between two outcomes [11]. Pair sample is commonly used in case-control studies and is often used in experiments where two observations of before and after condition are used to compare. In this paper, the two-sample t-test is applied since the goal is to validate whether the condition that the damping rate of designed PSS is significantly different from the damping measurements of conventional tuned PSS.

The null hypothesis assumes that the true mean difference between the paired samples is zero. Conversely, the alternative hypothesis assumes that the true mean difference between the paired samples is not equal to zero. The paired sample t-test hypotheses are formally defined [12] as:

- The null hypothesis $\left(\mathrm{H}_{0}\right)$ assumes that the true mean difference, $\mu_{\mathrm{d}}$ is equal to zero, and

- The alternative hypothesis $\left(\mathrm{H}_{1}\right)$ assumes that $\mu_{\mathrm{d}}$ is not equal to zero.

\subsection{Paired sample t-test}

When the samples are dependent, a paired sample t-test for dependent means is used. This test employs the difference in values of the matched pairs [13]. Firstly, it is required to find the differences in the values of the sample pairs of data and it is denoted by (7).

$$
D=\bar{X}-\bar{Y}
$$

After calculating D, the sum of the difference scores $(\Sigma D)$, the sum of the squared difference scores $\left(\Sigma \mathrm{D}^{2}\right)$, and the mean difference score $\overline{\mathrm{D}}$ and the standard deviation of the difference scores $\left(\mathrm{s}_{\mathrm{D}}\right)$ are calculated, respectively. The standard deviation $\left(\mathrm{s}_{\mathrm{D}}\right)$ of the samples is defined by (8) and (9).

$$
S_{D}=\sqrt{\frac{S S_{D}}{n-1}}
$$

where,

$$
\mathrm{SS}_{D}=\sum_{i=1}^{n} D_{i}^{2}-\frac{\left(\sum_{i=1}^{n} D_{i}\right)^{2}}{n}
$$

The test value $\mathrm{t}$ formula is shown in (10) with the degree freedom (d.f), $\mathrm{n}-1$.

$$
t=\frac{\bar{D}-\mu_{D}}{\frac{s_{D}}{\sqrt{n}}}
$$

Finally, it is required to evaluate the t-obtained value based on alpha $(\alpha)=0.05$ and to compute the probability of the mean differences of the samples under the null hypothesis, $\mu_{D}=0$.

\section{2. t-test procedure and result interpretation}

The procedure for a paired sample t-test consists of four steps. The first one is calculating the sample mean and the sample standard deviation. And then, the test statistic is tabulated. Finally, the probability (p) of observing the test statistic under the null hypothesis is carried out. This value is obtained by comparing $\mathrm{t}$ to a t-distribution with $(\mathrm{n}-1)$ degrees of freedom. This process can be done by statistical software for more accurate results. When interpreting the results of a paired sample t-test, statistical significance is determined by looking at the p-value. The lower the p-value, the lower the probability of obtaining a result like the one that was observed if the null hypothesis was true [9]. Therefore, to perform the t-test, the damping ratios of the power system with PSSs are used as the sample data of t-test. These data are analyzed in the following section. 


\section{DETECTION AND ANALYSIS OF OSCILLATION MODES}

In this section, the previous research [4], [6] observation is utilized to confirm these results by applying t-test. In [4], [6], a practical model of the MEPE test system was used to validate the performance of PSS for damping oscillation. The total power generation is $3040 \mathrm{MW}$ and $1120 \mathrm{MVAr}$ while the total real power demands of the system are $2727 \mathrm{MW}$ in the MEPE test system. The overall system information is provided in [4]. The stability analysis of the MEPE test system with and without PSSs are observed and analyzed using free and open-source software PSAT [14]. Based on the results of [4], [6], three cases; MEPE test system without PSS, with tuned PSS and with designed PSS are considered and these results are used as two sample inputs for t-test.

For the case study, the small-signal analysis routine of PSAT [14] was used for detecting the lowfrequency oscillation modes. The complex eigenvalues $(\lambda=\sigma \pm j \omega)$ occur in conjugate pairs and the nonzero eigenvalues, $( \pm j \omega)$ define the electromechanical modes. The damping ratio is a good measure for damping [15]. The damping coefficient $\left(\zeta_{\mathrm{i}}\right)$ and oscillation frequency $\left(\mathrm{f}_{\mathrm{i}}\right)$ of the $\mathrm{i}^{\text {th }}$ eigenvalue are [16] defined as (11) and (12).

$$
\begin{aligned}
& \xi_{i}=\frac{-\sigma_{i}}{\sqrt{\sigma_{i}{ }^{2}+\omega_{i}{ }^{2}}} \\
& f=\frac{\omega}{2 \pi}
\end{aligned}
$$

In the power system, electromechanical oscillations with damping ratios greater than 0.03 are considered as a reasonable level [17]. A damping ratio between $5 \%$ to $10 \%$ is acceptable for secure system operation; however, the $10 \%$ value is recommended for interconnected systems [18]. The current trend is to require the damping ratio to be higher than 0.05 for all modes overall operating conditions [6].

\subsection{Damping influences of PSS in MEPE test system}

In the MEPE test system, there are 40 generators and the numbers of state variables for the dynamic model depend on the type of generations. This paper briefly explains the eigenvalues result and the detailed theory and results are presented in [4], [6]. Through the dynamic analysis pane of PSAT [19], the plots of eigenvalues of the MEPE test system without and with PSS are illustrated in Figure 1 and Figure 2 respectively.

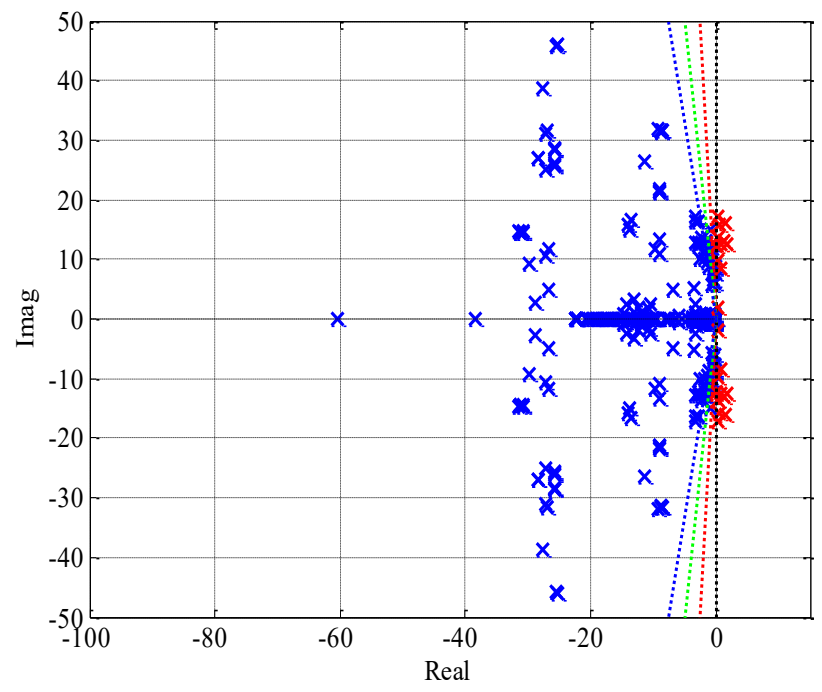

Figure 1. Eigenvalue analysis of MEPE test system

The eigenvalues indicate the system stability and how close the system is to become unstable. It also shows what frequencies and oscillation modes exist in the system [20]. According to the result of Figure 1, the critical modes with their oscillation frequency, damping ratio, and nature of the modes of MEPE test system have been achieved based on (11) and (12). From these results, three of these low-frequency modes present a damping ratio lower than $3 \%$ and the modes with less than $5 \%$ are presented in prior research [4]. 


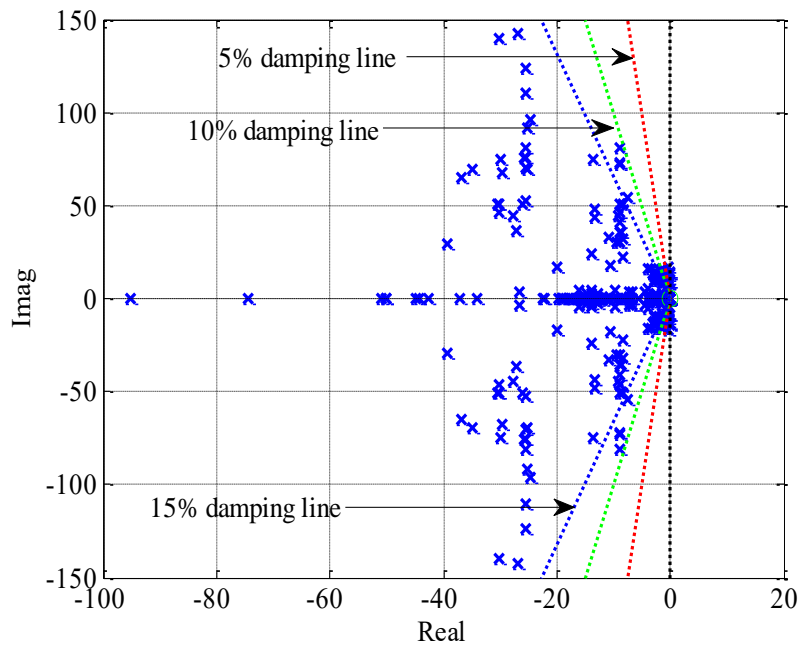

Figure 2. Eigenvalue analysis of MEPE test system with PSS

To improve the damping, PSS is installed on the dominant generator and the resultant eigenvalues of the MEPE test system with PSSs are displayed in Figure 2. The positive eigenvalues (redpoint) in Figure 1 are shifted to the left that can be seen in Figure 2. After installing the PSS, all eigenvalues are negative and damping ratios are higher than that of without installing condition.

\subsection{Investigation the damping ratio after installing designed PSS}

To achieve the best damping $(\zeta>0.05)$, the PSS parameter is designed based on the GEP method and this designed PSS is installed on dominant generators that are most influenced in oscillation [6]. The eigenvalues result of the MEPE test system with designed PSS is represented in Figure 3. This section points out the result only and the detailed design procedure and results are illustrated in [6].

From the result of Figure 3, the damping ratios of all low-frequency modes are greater than $5 \%$ except Mode 15 that are denoted by oscillation frequency of eigenvalues result. The damping ratio of the MEPE test system is compared before PSS installation, after placing manual tuned PSS, and after installing the designed PSS. According to the eigenvalues results, the damping ratios and associated generators of low frequency modes are pointed out. There are 20 machines out of 40 that are sensitively influenced on damping and the relative damping ratios of three conditions are compared in Figure 4.

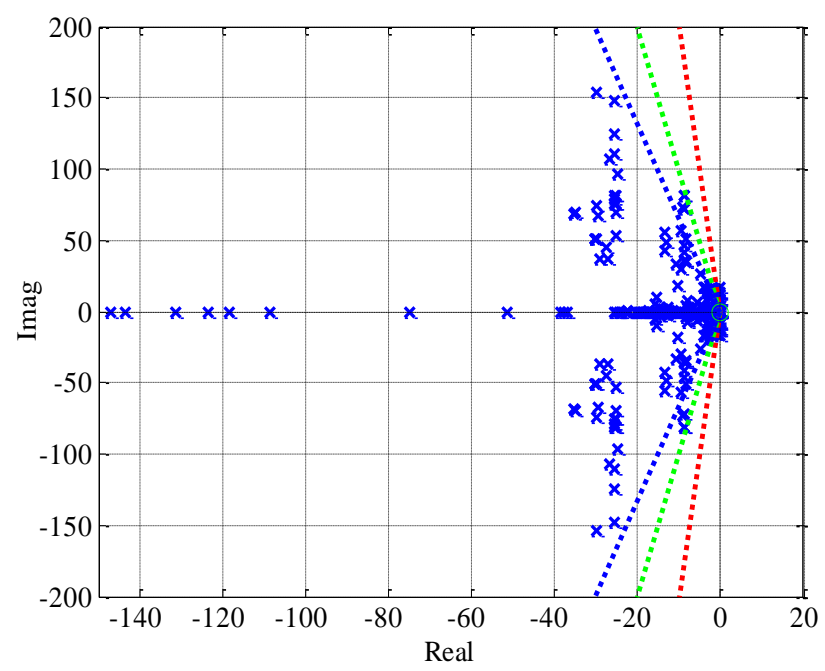

Figure 3. Eigenvalue result of MEPE test system with designed PSS 


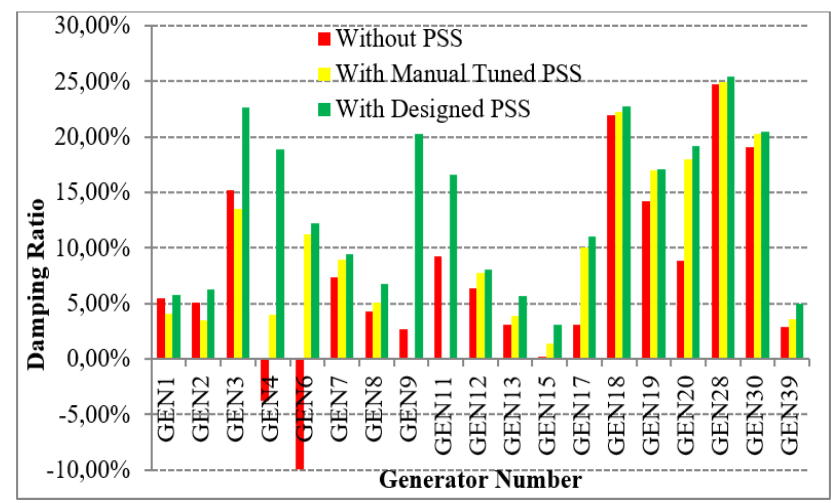

Figure 4. Comparative result of damping ratio for MEPE test system

It can be observed from Figure 4 that the damping ratio of G4 and G6 are negative before installing PSS. Therefore, the damping ratio of the MEPE test system is increased after installing PSS when compared to the negative damping (G4 and G6) before installing PSS. Besides, the damping rates of the MEPE test system with design PSS are significantly uppermost in all conditions, and all damping ratios are reached to stability region with the values of greater than $5 \%$ except G15. The overall damping ratio of the MEPE test system is significantly improved when installing the PSS designed based on the GEP method. In the next section, a statistical hypothesis test is used to prove the effect of designed PSS.

\section{STATISTICAL t-TEST FOR LOW-FREQUENCY OSCILLATION DAMPING}

To evaluate the effectiveness of PSS, the statistical t-test is applied in this section. To prove with a t-test, two samples (or treatments) are required. The data input for the t-test is damping ratios of the test system because it is needed to assess the impacts of PSS and to make the decision that PSS is more effective. As presented in section 4, the damping ratios of the respective situation are organized as the input parameters of the statistical t-test. In Table 1, damping ratios of three cases (without PSS, with PSS, and with designed PSS) with the same modes are planned. Theses damping ratios are used as the input parameters for statistical t-test.

Table 1. Input parameters of statistical t-test

\begin{tabular}{ccccc}
\hline \multicolumn{5}{c}{ Input Parameters of Statistical t-Test } \\
Sr. No. & Mode No. & Without PSS & With PSS & With designed PSS \\
\hline 1 & 1 & $-12.62 \%$ & $22.29 \%$ & $12.22 \%$ \\
2 & 2 & $-5.99 \%$ & $22.27 \%$ & $22.59 \%$ \\
3 & 3 & $5.06 \%$ & $3.54 \%$ & $22.25 \%$ \\
4 & 5 & $0.08 \%$ & $17.97 \%$ & $18.90 \%$ \\
5 & 6 & $8.81 \%$ & $12.81 \%$ & $20.29 \%$ \\
6 & 7 & $17.18 \%$ & $4.08 \%$ & $19.19 \%$ \\
7 & 8 & $5.50 \%$ & $18.15 \%$ & $22.65 \%$ \\
8 & 10 & $6.35 \%$ & $7.65 \%$ & $25.43 \%$ \\
9 & 11 & $19.07 \%$ & $20.29 \%$ & $20.45 \%$ \\
10 & 12 & $-3.28 \%$ & $11.15 \%$ & $8.01 \%$ \\
11 & 13 & $2.87 \%$ & $6.04 \%$ & $20.43 \%$ \\
12 & 16 & $-9.98 \%$ & $3.35 \%$ & $4.56 \%$ \\
13 & 17 & $3.13 \%$ & $16.91 \%$ & $17.11 \%$ \\
14 & 18 & $4.26 \%$ & $4.37 \%$ & $5.69 \%$ \\
15 & 19 & $2.64 \%$ & $4.93 \%$ & $6.78 \%$ \\
16 & 20 & $7.36 \%$ & $7.95 \%$ & $9.46 \%$ \\
17 & 21 & $3.12 \%$ & $0.51 \%$ & $11.05 \%$ \\
18 & 23 & $57.30 \%$ & $95.80 \%$ & $95.87 \%$ \\
19 & 25 & $97.11 \%$ & $90.84 \%$ & $91.23 \%$ \\
21 & 28 & $80.93 \%$ & $98.59 \%$ & $98.83 \%$ \\
21 & 29 & $97.88 \%$ & $88.63 \%$ & $98.63 \%$ \\
\hline & & & &
\end{tabular}

The problem desires a $5 \%$ level of significance which equates to a $95 \%$ level of certainty. Therefore, the significance level $\alpha=0.05$ is established for $95 \%$ confidence interval and the degrees of 
freedom (df) is (n-1). As presented in section 3.2, required t-test parameters are calculated and the results including the value of $\mathrm{t}$ and $\mathrm{p}$ are reported in the table.

For evaluating the effectiveness of PSS for power system oscillation damping, a suitable null hypothesis would be that there is no difference in the damping ratio between the two conditions.

Let the null hypothesis: $\mathrm{H}_{0}=\mu_{2}-\mu_{1}=0$.

Let the alternative hypothesis: $\mathrm{H}_{1}=\mu_{2}-\mu_{1}>0$.

\subsection{Without PSS versus with PSS}

Firstly, the damping effects of without PSS and with PSS are evaluated using t-test in this section. With the providing of eigenvalue analysis, the damping of two scenarios namely: Without PSS and with PSS at same modes are selected as two data set inputs for t-test. This test decides that

"Is there a specific difference between with and without PSS or not?"

where, $\mu_{1}$ and $\mu_{2}$ are the mean values of the damping ratio of without and with PSS respectively. The output parameters of the statistical t-test are shown in Table 2.

Table 2. Output parameters of statistical t-test for case I

\begin{tabular}{lll}
\hline \multicolumn{3}{c}{ t-Test: Paired Two Sample for Means } \\
& Without PSS & With PSS \\
\hline Mean & 0.219968182 & 0.298354545 \\
Variance & 0.13753418 & 0.133094775 \\
Observations & 21 & 21 \\
Pearson Correlation & 0.932254673 & \\
Hypothesized Mean Difference & 0 & \\
df & 20 & \\
t Stat & -2.712834823 \\
P(T<=t) one-tail & 0.00651669 & \\
t Critical one-tail & 1.720742903 & \\
P(T<=t) two-tail & 0.01303338 & \\
t Critical two-tail & 2.079613845 & \\
\hline
\end{tabular}

It can be seen from the result that the means are significantly different since the p-value is extremely low. The means for the damping ratio are significantly different at $\mathrm{p}=0.00651669$. Therefore, it can be practically assured that the mean of with PSS is greater than those of the test system without PSS. The result decides to reject the null hypothesis. It can be depicted that the results of the MEPE test system with PSS is more suitable than that of without PSS.

\subsection{Without PSS versus with designed PSS}

The two data set inputs for t-test are the damping ratio of case II: Without PSS and with designed PSS. This test decides that

"Is there a specific difference between without PSS and with designed PSS or not?"

The null hypothesis and alternative hypothesis are defined as the earlier section. Where, $\mu_{1}$ and $\mu_{2}$ are the mean damping ratio of without PSS and with designed PSS. The results of the statistical t-test are exposed in Table 3.

Table 3. Output parameters of statistical t-test for case II

\begin{tabular}{lcc}
\multicolumn{3}{c}{ t-Test: Paired Two Sample for Means } \\
& Without PSS & With Designed PSS \\
\hline Mean & 0.184180952 & 0.310288762 \\
Variance & 0.114826124 & 0.108729923 \\
Observations & 21 & 21 \\
Pearson Correlation & 0.948643484 \\
Hypothesized Mean Difference & 0 \\
df & 20 \\
t Stat & -5.374946208 \\
P (T<=t) one-tail & 0.0000146048 \\
t Critical one-tail & 1.724718243 \\
P(T<=t) two-tail & 0.0000292096 \\
t Critical two-tail & 2.085963447 \\
\hline
\end{tabular}


Among the variables that appear in the results table, depending on the research purpose, the p-value is the most important. Since the value of $\alpha$ is set at 0.05 and the p-value (0.0000146048) is expressively less than 0.05 , it can be said that there is a statistically significant difference. Also, the mean of two data sets is different, the null hypothesis is rejected. Therefore, the second case (with designed PSS) is more effective for system damping.

\subsection{With PSS versus with designed PSS}

In this section, the MEPE test system with PSS and designed PSS are assessed with a statistical test to determine whether the PSS is more effective. These data inputs are demonstrated in Table 1. To decide the specific differences between the two PSS, the hypothesis question is that

"Is there is a specific difference between with PSS and designed PSS or not".

where, $\mu_{1}$ and $\mu_{2}$ are the mean damping ratio of case III: Test system with PSS and with designed PSS.

The t-test results are in the Table 4. From the results, the p-value of both one tail $(0.006059454)$, and two tail (0.012118907) are less than 0.05 of the desired confidence intervals. The means are significantly different because the probability is low. The mean of the first situation (with PSS) is less than that of the second situation (with designed PSS). Therefore, the decision is the power to reject the null hypothesis. It can be concluded from the test results that the designed PSS is more effective than that of tuned PSS.

Table 4. Output parameters of statistical t-test for case III

\begin{tabular}{lcc} 
& t-Test: Paired two sample for means \\
& With PSS & With Designed PSS \\
\hline Mean & 0.265771429 & 0.310288762 \\
Variance & 0.115225179 & 0.108729923 \\
Observations & 21 & 21 \\
Pearson Correlation & 0.975988219 \\
Hypothesized Mean Difference & 0 \\
df & 20 \\
t Stat & -2.758447129 \\
P $(T<=t)$ one-tail & 0.006059454 \\
t Critical one-tail & 1.724718243 \\
P $(T<=t)$ two-tail & 0.012118907 \\
t Critical two-tail & 2.085963447 \\
\hline
\end{tabular}

\subsection{Overall discussion on statistical t-test}

The parametric test called t-test is used for conducting statistically significant tests in the testing hypotheses. The $\mathrm{t}$ value will be positive if the first mean is larger than the second and negative if it is smaller. In this test, it is needed to take 0.05 as the appropriate level of significance while conducting the t-test. The level of significance refers to the minimum probability that there will be a false rejection of the null hypothesis. If the calculated $\mathrm{p}$ value is less than 0.05 , the conclusion is that, statistically, the mean difference between the paired observations is significantly different. The probability results are expressed in Figure 5.

P Value (one-tail) of t-Test for Without PSS versus Tuned PSS

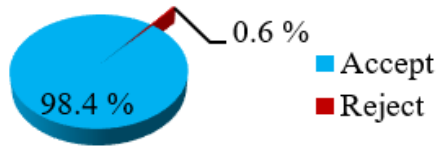

P Value (two-tail) of t-Test for Without PSS versus Tuned PSS

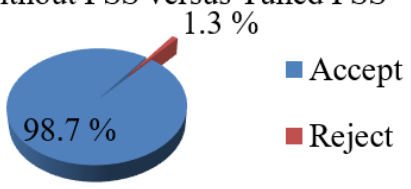

(a)
$P$ Value (one-tail) of t-Test for Without PSS versus Designed PSS

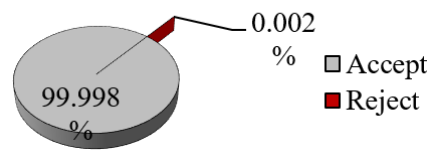

P Value (two-tail) of t-Test for Without PSS versus Designed PSS

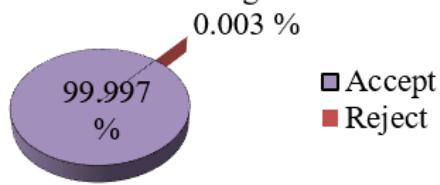

(b)
P Value (one-tail) of t-Test for Tuned PSS versus Designed PSS

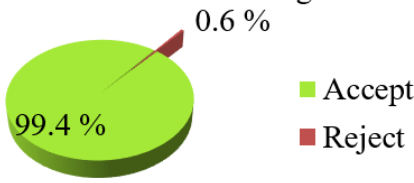

P Value (two-tail) of t-Test for Tuned PSS versus Designed PSS

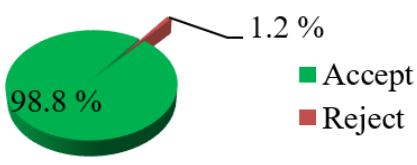

(c)

Figure 5. Probability results of statistical t-test: (a) Without PSS vs tuned PSS, (b) Without PSS vs designed PSS, (c) Tuned PSS vs designed PSS 
The results from Figure 5 show that the p value is significantly low and especially p values are about three in five hundred for Figure 5(a) and(c) and the probability of about seven in five hundred thousand. It can be said that the null hypothesis gets rejected and the MEPE test system with designed PSS is most applicable for the improvement of oscillation damping in the power system in all statistical t-tests.

\section{CONCLUSION}

In this paper, a statistical t-test is applied to monitor and assess the different performances of PSS and to certify the effectiveness of designed PSS. To improve the stability and to achieve better damping oscillation, manual tunned PSS and designed PSS are equipped on the generator of the MEPE test system is presented. Eigenvalues analysis is provided to demonstrate the damping ratio and low-frequency modes of the associated generator. PSS based design methodology is provided to improve the oscillatory stability. This paper has proposed the statistical test to assess the damping rate of PSS for power system oscillation. The concept of hypothesis has been explained as the difference in the application of tuned PSS and designed PSS. The null hypothesis, $\mathrm{H}_{0}$ was that there is no significant difference in damping ratio means with the use of designed PSS. The t-test result showed that there is a significant difference in means of damping ratios with the application of designed PSS and is encouraged to be accepted by applying designed PSS. Based on the probability value of statistical t-test analysis, the designed PSS achieved the targeted stability margins and enhances the damping of low-frequency modes significantly at a 95 percent confidence interval.

\section{REFERENCES}

[1] F. Yang-chun, H. Yan-chun and H. Yan-chun, "The application of student's t-test in internal quality control of clinical laboratory," Frontiers in Laboratory Medicine, vol. 1, no. 3, pp. 125-128, 2017, doi: 10.1016/j.flm.2017.09.002.

[2] D. Mondal, A. Chakrabarti and A. Sengupta, Power system small signal stability analysis and control, Elsevier Inc. 2014.

[3] K. M. Lin, W. Swe and P. L. Swe, "Open source software based modeling of MEPE test system for stability studies," International Journal of Energy and Power Engineering, vol. 4, no. 1, pp. 23-31, 2015, doi: 11648/j.ijepe.20150401.14.

[4] W. M. Thu and K. M. Lin, "Mitigation of low frequency oscillations by optimal allocation of power system stabilizers: Case study on MEPE test system," Energy and Power Engineering, vol. 10, no. 8, pp. 333-350, 2018, doi: 10.4236/epe.2018.108021.

[5] P. Dey, A. Bhattacharya and P. Das, "Tuning of power system stabilizer for small signal stability improvement of interconnected power system," Applied Computing and Informatics, vol. 16, no. 1/2, pp. 3-28, 2018, doi:10.1016/j.aci.2017.12.004.

[6] W. M. Thu and K. M. Lin., "Composite-design of power system stabilizer for multi-machine power system using gep and frequency response method," Journal of Research \& Innovation (JRI), vol. 3, no. 2, pp. 16-21, 2020.

[7] E. McCrum-Gardner, "Which is the correct statistical test to use?," British Journal of Oral and Maxillofacial Surgery, vol. 46, no. 1, pp. 38-41, 2008, doi: 10.1016/j.bjoms.2007.09.002.

[8] W. C. Sant'Ana, et al., "On the use of hypothesis tests as statistical indexes for frequency response analysis of electric machinery," Electric Power Systems Research, vol. 147, pp. 245-253, 2017, doi: 10.1016/j.epsr.2017.03.005.

[9] S. M. Ross, Introduction to probability and statistics for engineers and scientists, USA: Elsevier Inc, 2004.

[10] S. Glen, "Correlation coefficient: Simple defination, formula, easy steps," [Online]. Available: https://www.statisticshowto.com/probability-and-statistics/correlation-coefficient-formula/.

[11] J. Kaur, "Techniques used in hypothesis testing in research methodology a review," International Journal of Science and Research (IJSR), vol. , no. 54, pp. 362-365, 2015.

[12] R. R. Wilcox, Introduction to Robust Estimation and Hypothesis Testing, USA: Elsevier Inc., 2005.

[13] T. H. Abebe, "The derivation and choice of appropriate test statistic (Z, t, F and Chi-Square Test) in research methodology," Mathematics Letters. vol. 5, no. 3, pp. 33-40, 2019, doi: 10.11648/j.ml.20190503.11.

[14] F. Milano, Power system analysis toolbox documentation for PSAT, version 2.1.8, Jan. 6, 2013.

[15] W. Du and H. Wang, Analysis and damping control of power system low frequency oscillations, USA: Springer, 2016.

[16] M. J. Gibbard, P. Pourbeik and D. J. Vowles, Small signal stability, control and dynamic performance of power systems, Adelaide: University of Adelaide Press, 2015.

[17] J. Machowski, Z. Lubosny, J. W. Bialek, and J. R. Bumby, Power system dynamic and stability, 2nd ed, UK: John Wiley \& Sons Ltd, 2017.

[18] G. Rogers, Power system oscillations, USA: Springer, 2000, doi:10.1007/978-1-4615-4561-3.

[19] F. Milano, "An open source power system analysis toolbox," in IEEE Transactions on Power Systems, vol. 20, no. 3, pp. 1199-1206, Aug. 2005, doi: 10.1109/TPWRS.2005.851911.

[20] K. Prasertwong, N. Mithulananthan and D. Thakur, "Understanding low frequency oscillation in power systems," International Journal of Electrical Engineering Education, vol. 47, no. 3, pp. 248-262, 2010, doi: 10.7227/IJEEE.47.3.2. 


\section{BIOGRAPHIES OF AUTHORS}

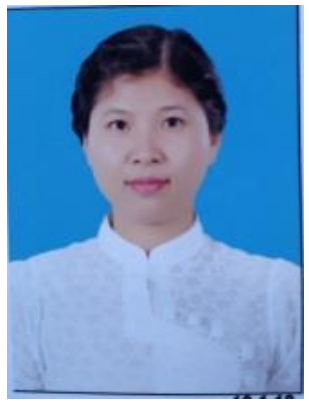

Wai Myat Thu received her B.E degree in Electrical Power Engineering from Technological University of Hinthada, Myanmar in 2007, and M.E and Ph. D degree in Electrical Engineering from Mandalay Technological University (MTU), Mandalay, Myanmar, in 2011 and 2018, respectively. She has researched the Power System Research Unit of MTU under the supervision of Dr. Kyaw Myo Lin till 2018. She is currently a lecturer at Technological University (Thanlyin). Her research interests are power system stability and control.

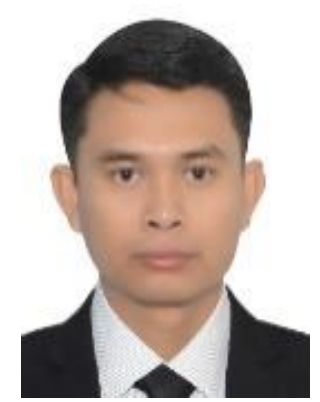

Kyaw Myo Lin received his $\mathrm{Ph}$. D degree in Electrical Engineering from Mandalay Technological University (MTU), Mandalay, Myanmar, in 2014. After that, he set up the Power System Research Unit at MTU and has done the research area of power system stability and optimization as well as reliability and energy management of the distribution system. Now, he is the professor and head of the Department of Electrical Power Engineering at Pyay Technological University, Pyay, Myanmar. 